\title{
Hypolipemic and weight reducing properties from Tamarindus indica L. pulp extract in diet-induced obese rats.
}

\begin{abstract}
The global prevalence of overweight and obesity has reached epidemic proportions. Hyperlipemia couple with increased oxidative stress generates various degenerative diseases such as hypertension and cardiovascular problems. In the present study, a hypolipemic and weight reducing effects of crude Tamarindus indica L. pulp extract were examined in adult Sprague-Dawley rats fed a high-fat diet. Animals were fed on either normal chow or high-fat diet for 10 weeks for obesity induction and subsequently received either placebo or T. indica L. extract at $5,25,50$ or $300 \mathrm{mg} \mathrm{kg}-1$ chitosan via oral gavage for another 10 weeks. Treatment of obese rats with the T. indica pulp extract led to a decrease in the levels of plasma total cholesterol (TCHOL), low-density lipoprotein cholesterol (LDL-C) and triglyceride (TG) and increase high-density lipoprotein cholesterol (HDL-C) level with concomitant reduction of body weight. The extract improved the efficiency of the antioxidant defense system, as indicated by increased superoxide dismutase (SOD) and glutathione peroxidase (GPx) activities and subsequently resulted in significantly lower lipid peroxidation indices; malondialdehyde (MDA) level. Together these results indicate the potential use of $\mathrm{T}$. indica extracts as hypolipemic and antioxidative agent apart from its ability to reduce body weight in obese-induced rats.
\end{abstract}

Keyword: Antioxidant; Free radical; Hypolipemia; Lipid peroxidation; Tamarind; Weight maintenance. 